Influence of Sodium Thiocyanate on the Development of the Chick Embryo

UNINCUBATED eggs were injected with $0.3-0.7 \mathrm{ml}$. of sodium thiocyanate of various concentrations $\left(0.1 M, 0.5, \frac{m}{M}, 1 M\right)$ and thiocyanate of various concentrations $(0 \cdot 1 M, 0.5 M, 1 M$, $M$ and
incubated for $42-47$ hours. The embryos thus developed showed a number of anomalies, first of all in the prosencephalon and partly in the mesencephalon; the metencephalon and myelencephalon being in general, not so much affected. One of the most frequently observed a nomalies of the latter was due to the abnormal closing of the neural tube: it seems that one of the neural folds was growing more quickly, tube : it seems that one of the neural folds was growing more quickly, tended in various cases to the middle of the myelencephalon. Further tended in various cases to the middle of the myelencephalon. Further
changes were observed in the position of the heart, which was dis-
placed, in extreme cases, into the precephalic region. No morphoplaced, in extreme cases, into the precephalic region. No morpho-
genetical changes of the somites were observed, though the number was lower (14-17) than that of normal embryos of the same age.

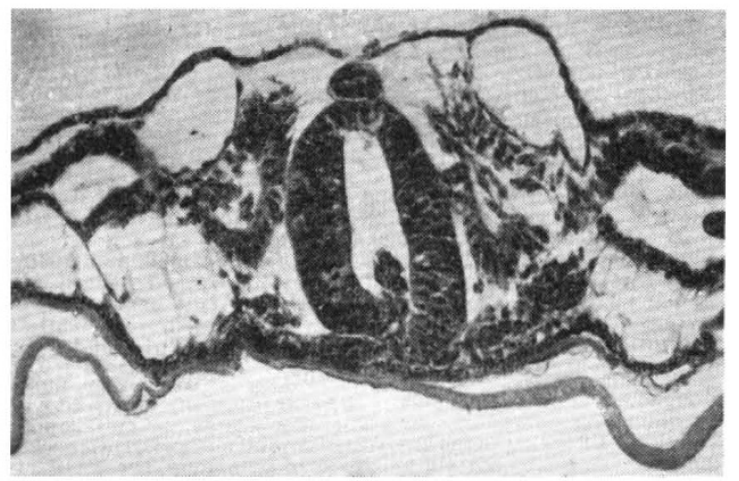

CHICK EMBRYO INJECTED WITH 0.3 ML. OF $M$ SODIUM THIOCYANATE. INCUBATION FOR 42 HOURS. TRANSVERSE SECTION AT THE LEVEI OF THE FIRST SOMITE.

The question whether the notochord was hyperdeveloped is still not very clear : in some cases it was certainly folded, but it is difficult for us to decide at the moment whether this is due to the same cause as described by Ranzi ${ }^{1}$ for the amphibian embryos.

In conclusion, we may say that sodium thiocyanate inhibits, but to various degrees, the growth of different organs. Here the heteroauxesis ${ }^{2,3}$ is particularly in evidence : for example, the brain in many cases corresponds to a much younger embryo than the number of
somites would suggest. Even inside one and the same organ we found somites would suggest. Even inside one and the same organ we found
differences in the growth-rate, as noted above in the growth of the differences in the growth-rate, as noted above in the growth of the
neural folds. The heart, in comparison with the rest of the body, also shows typical bradyauxesis.

From the morphogenetical point of view, sodium thiocyanate acts, flrst of all, on the brain. No definite influence, besides an inhibitory effect on the meso- and endoderm, could be observed.

Details, with biochemical analyses, will be published elsewhere.

Instituto de Anatomfa General y Embriología, TOR W. NOWINSKI. Facultad de Ciencias Médicas, Universidad de Buenos Aires. Aug. 25.

' Ranzi, S., Nature, 155, 578 (1945).

2 Needham, J., and I.erner, I. M., Nature, 146, 618 (1940).

Needham, J., "Biochemistry and Morphogenesis" (Cambridge University Press, 1942).

\section{Effect of Choline on the Intestinal Absorption of Fat}

THE effect of choline on intracellular fat in the liver and other organs has been extensively investigated. So far as we can discover, no experiments have been published on the effect of choline on the fat normally present in the intestinal cells during absorption.

Striking differences can be demonstrated between the distribution of fat in the intestine of rats fed 1 c.c. of olive oil and 1 c.c. of water and those fed 1 c.c. of olive oil and 1 c.c. of 0.5 per cent choline chloride. The difference can be seen in the accompanying photographs. After olive oil and water, the cells are flled with large globules of fat and very little appears to have passed through into the areolar tissue of the villus, or into the lacteals. When olive oil and choline solutions are similarly administered, and the animal examined after the same time period, masses of fat can be seen in the areolar tissue of the villus, and the cells are more rapidly cleared. The fat remaining in the cells appears to be more finely dispersed.

The effect is immediate, and occurs in animals without previous dietary restriction, except for the usual preparatory without previous period. The contrast is marked in spite of the period. The contrast is marked in spite of the probable presence of small amounts of choline derived from the bile and elsewhere in the control animals. This may be explained by the relatively large amount of olive oil used in these experiments (1 c.c.) as compared with the normal dietary fat intake of these animals. The evidence at present or marked increase of intestinal movement with the doses of $2-5 \mathrm{mgm}$. of choline which have been used in these studies.
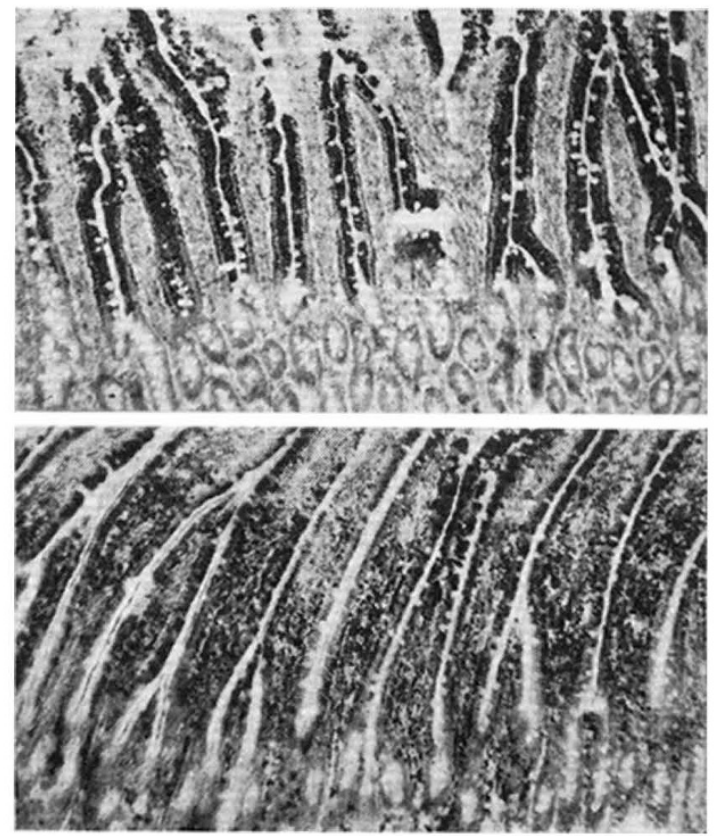

PHOTOMICROGRAPHS OF THE JEJUNUM $(\times 70)$ OF THE RAT: ABOVE, AFTER FEEDING 1 c.C. OLIVE OII AND 1 c.C. DISTIILED WATER; BELOW, AFTER FEEDING 1 c.c. OLIVE OIL AND 1 c.c. 0.5 PER CENT CHOIINE CHLORIDE SOLUTION. ANIMALS KILLED 3 HR. AFTER FEEDING BY GASTRIC TUBE. WISTAR STRAIN MALE RATS, AVERAGE WEIGHT 200 GM. STAIN, SCDAN IY AND HAMATOXYLIN.

The mechanism of this action of choline, and its possible relationship to normal fat absorption and to other lipotropic effects. are being investigated, and full experimental details will he published in due course.

Department of Pharmacology,

A. C. FRAJER.

University of Birmingham. Nov. 30 .

\section{$T$ Coronæ Borealis}

THE recent outburst of the ex-nova, $T$ Coronæ Borealis, first reported on February $9^{1}$, when its magnitude was estimated to be $3 \cdot 4$, as against 11.3 during the immediately preceding epoch ${ }^{2}$, has been observed at Sidmouth on three occasions only, owing to unfavourable weather conditions. Three objective-prism spectrograms of the star, secured with the 12-in. McClean prismatic camera, form the total photographic observations to date ; in addition, four visual estimates of brightness were made by the aid of fleld-glasses.

The first observation was made through drifting cloud on the early morning of February 11 at $05 \mathrm{hr}$. C.T. At that time the visual magnitude was estimated as $3 \cdot 3$. The spectrogram, much under-exposed, shows little structure other than the prominent Balmer emissions at $\lambda \lambda 4340$ 4861 , and the bright bands at $\lambda \lambda 4640,4686$ due to $N$ III $+O$ II, and $\mathrm{He}$ II respectively. The two latter features are somewhat better deflned than the Balmer lines, which are broad and diffuse. The order of intensities, as measured visually, is He II $>\mathrm{H}_{\gamma}>\mathrm{N}$ III +0 II. $>$ $\mathrm{H}_{\beta} . \mathrm{H}_{\delta}$ is only just discernible.

Persistently clouded skies prevented further observation until the night of February 19-20, when the star was seen for a brief interval through a cloud-gap, and its magnitude estimated to be somewhat less than $7 \cdot 0$

A second photograph was secured on the morning of March 3, by which time the star had faded still further to an estimated magnitude of $9 \cdot 0$. Owing to poor atmospheric transparency, and the intrinsic faintness of $T$ Coronæ, it was not possible to obtain a fully exposed spectrogram. However, the exposure allowable was sufficient to reveal the main post-maximum structure, and it was noted that again, the He II emission was the brightest feature of the entire spectrum, it having strengthened considerably. This latter observation was confirmed from an examination of the most recent photograph secured on March 10, when the stellar brightness had faded to about mag. $10 \cdot 0$.

No estimates of the velocity of expansion of the ejected shell have been possible on account of the poor quality of the spectrograms.
D. R. BARBER.

Norman Lockyer Observatory, Sidmouth, Devon. March 13.

${ }^{1}$ See Nature, 157, 224, 261, 270 (1946)

${ }^{2}$ Reported by Miss F. W. Wright, Harvard Observatory. I.A:U. Circular No. 1030. 